\title{
Research Concerning the Ramp and Sinusoidal Command Signals of the Piezoelectric Miniactuators
}

\author{
Simona NOVEANU ${ }^{1, \text { a }}$, Dan MANDRU ${ }^{1, b}$ Alexandru IVAN ${ }^{2, c}$ \\ and Vencel CSIBI ${ }^{1, d}$ \\ ${ }^{1}$ Technical University of Cluj-Napoca, Romania \\ ${ }^{2}$ FEMTO-ST Institute, AS2M Department, France \\ a'Simona.Noveanu@mmfm.utcluj.ro, ${ }^{b}$ Dan.Mandru@mmfm.utcluj.ro, ${ }^{c}$ alex.ivan@femto-st.fr \\ dcsibiven@yahoo.com
}

Keywords: mini-actuator, piezoelectric, mini-system.

\begin{abstract}
In this paper is presented a mini-system with compliant minigripper and piezoelectric actuation. The piezoelectric actuator is controlled with ramp and sinus signals. The response of the mini-system is predicted by the finite elements method. The displacement of the compliant minigripper and piezoactuator are analyzed. In the end is presented a comparison of the experimental results for same actuator but for two different types of the signals with theoretical results.
\end{abstract}

\section{Introduction}

Mini-systems are diverse and complex and the trends indicate that it is appropriate to configure small-scale systems somewhat differently from those with which we are familiar. Simply downsizing from dimensions is not possible because of scaling effect. This is due to the fact that the types of forces that are dominant in miniaturized devices are different from those that are dominant in macro-scale devices. The designer of a mini-device needs to know the elastic properties in order to predict the amount of deflection from an applied force, or vice versa. If the material is ductile and the deformed structure does not need to return to its initial state, then the inelastic material behaviour is necessary. The strength of the material must be known so that the allowable operating limits can be set. The manufacturer of a mini and micro device needs to understand the relation between the processing and the properties of the material. New technologies tend to originate with new materials and manufacturing processes, which are used for new systems. The reduced dimensions involve different manufacturing technologies and special materials; measuring and control techniques and specific actuation methods based on new types of actuators [1].

The most representative fields the mini-systems applications are presented in figure 1 .

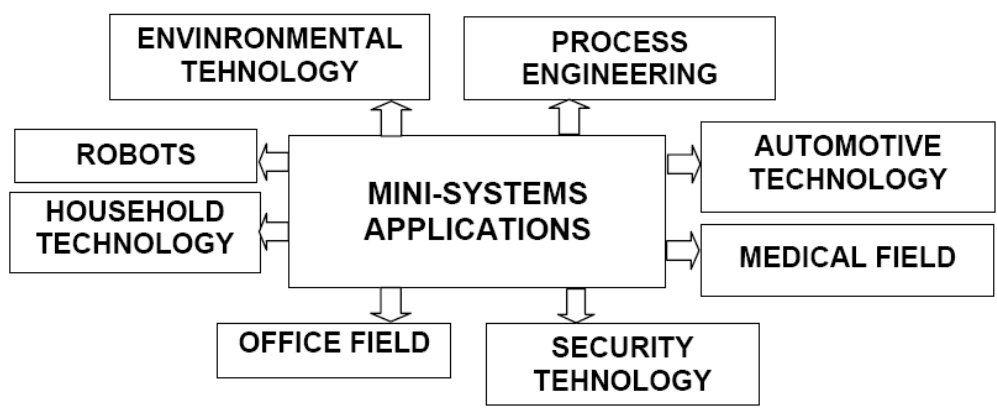

Fig.1 Mini-systems applications domains 


\section{Mini-actuators}

The mini systems have specific requirements to make them useful for handling miniature mechanical objects (shafts, bars, bearings, gears, wheels) or positioning different platforms with small dimensions. The actuation methods have a great influence on the overall performance of the mini-systems. The actuators convert an input energy (electrical, thermal, magnetic, chemical and optical) into controllable motion, based on the geometric characteristics of their mechanisms or on the material properties [2]. The mini-actuators for mini-systems include elements made of so called intelligent or smart materials (Fig. 2):

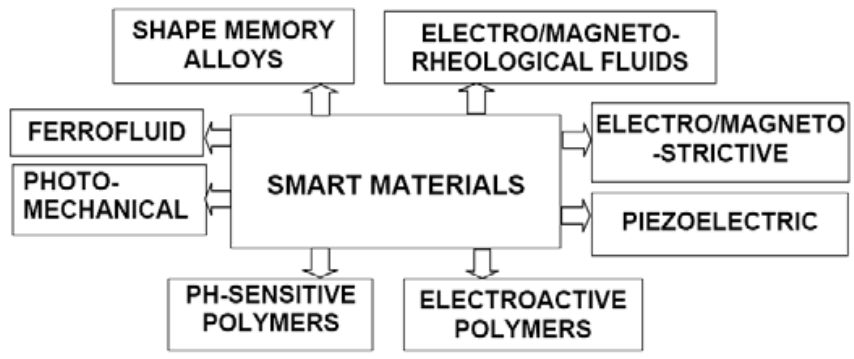

Fig. 2 Intelligent or smart materials

Regarding the piezoelectric materials, a tensile mechanical stress is induced that elongates and contracts the material due to the inverse piezoelectric effect, when they are placed in an alternative electric field. In order to amplify the actuators stroke, the stack and bimorph configurations are frequently used. Piezoceramic materials behave almost linear when the electromechanical loading is small. Below the Curie temperature they possess a spontaneous polarization, the direction can change when they are loaded in a high electromechanical field. This change in direction of the spontaneous polarization in the micro-structural level leads to a nonlinear behaviour of the material [3].

\section{Modelling of the Mini-system}

In the design of the mechanical structure of mini-systems based on compliant mechanisms, several advantages are considered: compact and monolithic construction, reduced wear and reduced need for lubrication, possibility of miniaturization [4].

The proposed mini-system has a mini-gripper actuated with a piezoelectric actuator (Fig. 3a). The gripper has a constant thickness denoted by $t$ and overall dimensions are presented in Fig.3b.

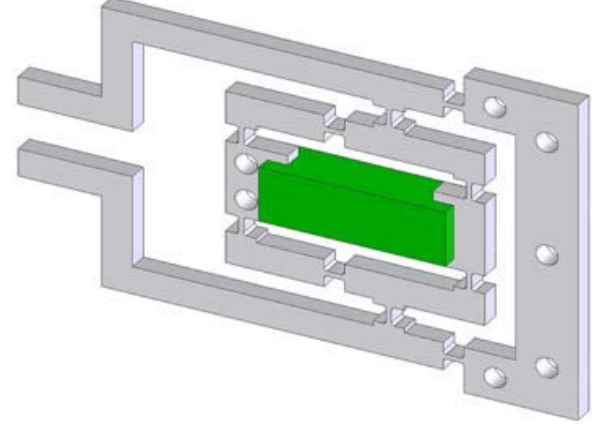

a

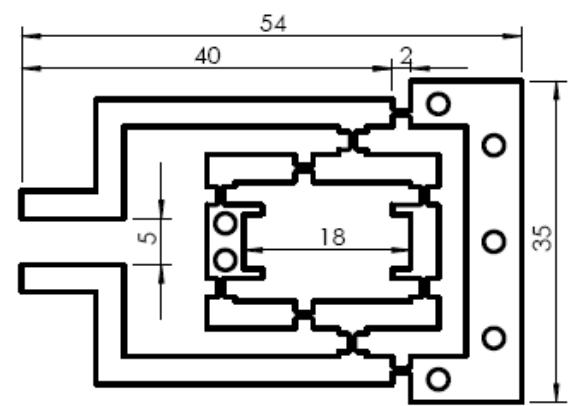

$\mathrm{b}$

Fig. 3 Mini-gripper with piezoelectric actuator (a) and overalls dimensions (b) 
Equations of the piezoactuator. The stack piezoelectric actuators have overall dimensions $18 \times 6.5 \times 6.5 \mathrm{~mm}$ and the following characteristics: maximum drive voltage $150 \mathrm{~V}$; capacitance $1600 \div 320 \mathrm{nF}$; clamping force $853 \mathrm{~N}$; resonant frequency: $261 \mathrm{KHz}$; Young's modulus $4.4 \times 10^{10}$ $\mathrm{N} / \mathrm{m}^{2}[5]$. The analytical equations for piezoelectric actuator displacements are:

$$
u_{G x}=(q U-F) / c_{T}
$$

Where $\mathrm{U}$ is voltage; $\mathrm{q}$ - force factors; $\mathrm{F}$ - axial force; $\mathrm{c}_{\mathrm{T}}$ - rigidity

The clamping force $\mathrm{F}_{0}$ is:

$$
F_{0}=q U_{\max }
$$

Equations of the minigripper. The gripper is built to enhance the input motion provided by the piezoelectric actuator. The ten flexure hinges are identical and have corner-filleted configuration [6]. The operation depends on the design of its mechanism which transmits the motion and force to the gripping fingers. The mechanical advantage can be found as the ratio of the input displacement, $\mathrm{u}_{\mathrm{Gx}}$, to the output displacement, $\mathrm{u}_{\mathrm{Hy}}$. The input displacement is determined by applying only the force $\mathrm{P}_{\mathrm{i}}$ at the input port by using Castigliano's theorem in the form:

$$
u_{G x}=\frac{\partial U}{\partial P_{i}}=\sum_{j=1}^{5}\left(\int_{0}^{l} \frac{N_{j}}{E A(x)} \frac{\partial N_{j}}{\partial P_{i}} d x_{j}+\int_{0}^{l} \frac{M_{j}}{E I(x)} \frac{\partial M_{j}}{\partial P_{i}} d x_{j}\right)
$$

Where $\mathrm{A}(\mathrm{x})=\mathrm{w}(\mathrm{x}) \mathrm{t}$ is the cross-sectional area and $\mathrm{I}(\mathrm{x})=\mathrm{tw}(\mathrm{x})^{3} / 12$ is the cross-sectional moment of inertia, $\mathrm{w}(\mathrm{x})$ being the variable width of the corner-filleted flexure hinge. It should be mentioned that the axial forces $\mathrm{N}_{\mathrm{j}}$ and bending moments $\mathrm{M}_{\mathrm{j}}$ start from actuator and reach to end effector. The $\mathrm{E}$ represents Young's modulus.

Because all the reaction forces and moments are only functions of $\mathrm{P}_{\mathrm{i}}$ and other length parameters of the device, the normal forces and bending moments are also going to depend on $\mathrm{P}_{\mathrm{i}}$.

The displacement at the output port under the action of $\mathrm{P}_{\mathrm{i}}$ can be calculated by using a force $\mathrm{P}_{\mathrm{o}}$ through:

$$
u_{H y}=\frac{\partial U}{\partial P_{o}}=\sum_{j=1}^{5}\left(\int_{0}^{l} \frac{N_{j}}{E A(x)} \frac{\partial N_{j}}{\partial P_{o}} d x_{j}+\int_{0}^{l} \frac{M_{j}}{E I(x)} \frac{\partial M_{j}}{\partial P_{o}} d x_{j}\right)
$$

In the end $\mathrm{P}_{\mathrm{o}}=0$. By combining eqs. (3) and (4), the mechanical advantage is obtained in the form:

$$
m \cdot a .=\frac{u_{H y}}{u_{G x}}
$$

The stiffness along the input direction is:

$$
k_{i}=\frac{P_{i}}{u_{G x}}
$$

In order to calculate the stiffness along the output direction, it is considered that only $\mathrm{P}_{\mathrm{o}}$ is acting at the output port. 
Simulation. The geometric parameters shown in Fig. 3 have been used to run the analytical and finite element simulation. It was considered the thickness of the gripper $\mathrm{t}=2.21 \mathrm{~mm}$.

The study was performed for the following inputs: voltage is $0 \div 150$ [V] and ramp signal. The Geometric Advantage resulted on the mini-gripper after FEM analyses are presented in the Fig. 4.

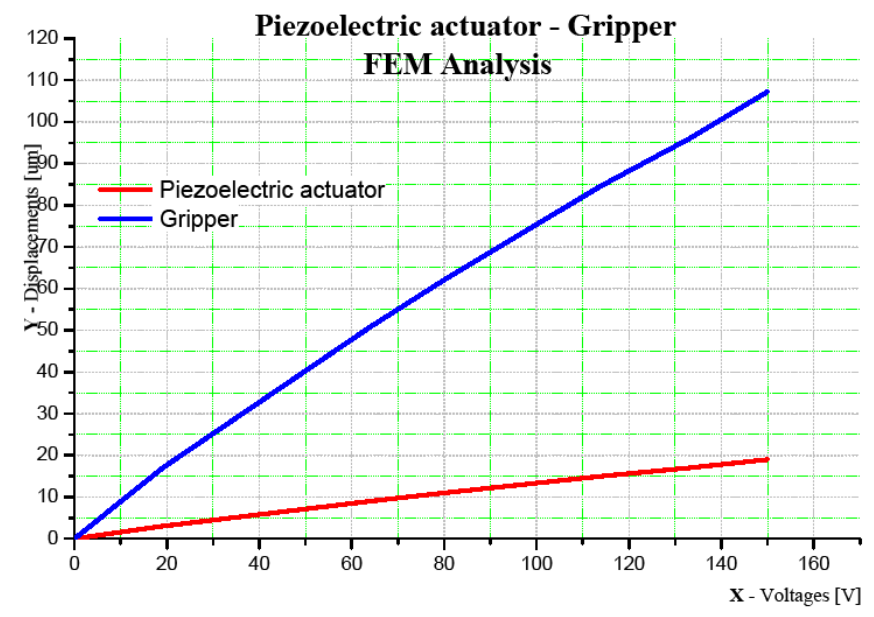

Fig. 4 Results for the FEM analyses

The material used for analysis of the mini-gripper is alloy steel with: $E=2.1 \times 10^{5} \mathrm{~N} / \mathrm{mm}^{2}$ and Poisson's Coefficient $=0.31$.

\section{Experimental Prototypes}

The design of complex flexible structures as well as the interactions at the system level between the: mechanical structure, actuators, sensors, electronics, control algorithms, allows, in fact, to benefit from the proposed compliant mechanisms approach in the design of mechatronic equipment.

A mini-system based piezo-actuated planar gripper is tested with regard to its mechanical advantage (amplification). The photograph of a gripper prototype is shown in Fig. 5a and the mini-system modules in Fig. 5b.

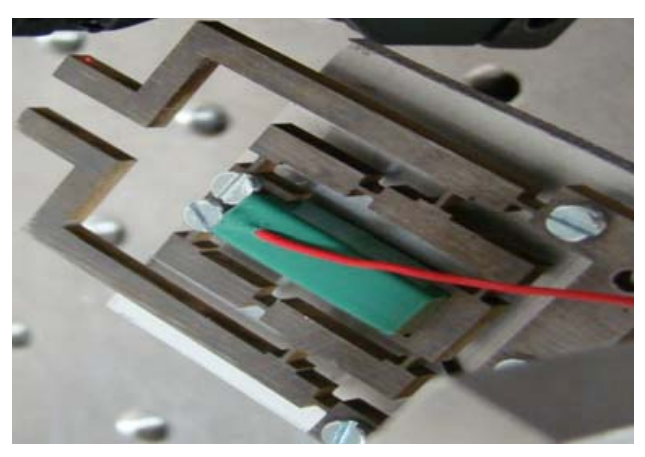

a

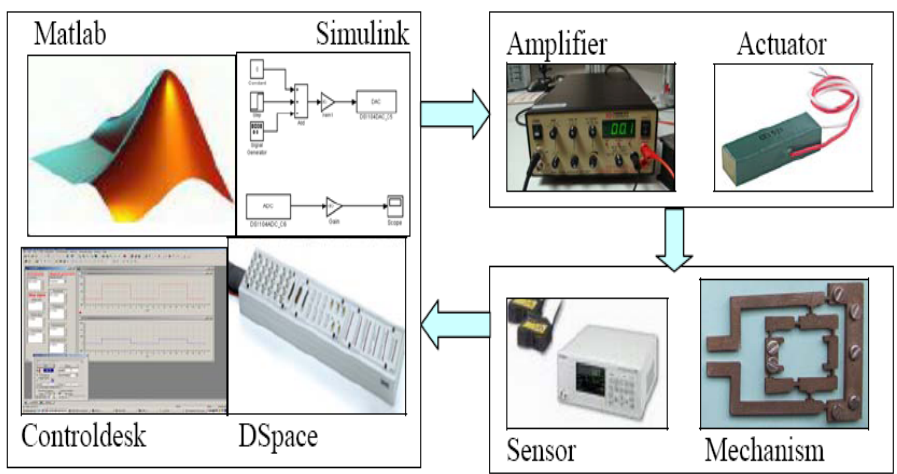

$\mathrm{b}$

Fig. 5 The photograph of a gripper prototype (a) and the mini-system modules (b)

For command and control of the mini-systems with data acquisition board DSpace, is made a Simulink model that generates the command signals using the Signal generator blocks. The signals type can be: step, sinusoidal, ramp, impulse, etc. Real-time view behaviour of the system will be 
through the Control Desk application. The control is achieved in closed loop, with a reaction by position; it is necessary to measure the actuators displacements using suitable position sensors; the control of supply voltage of actuators is such that it can be done the exact position required by the mechanism action. The structure of the test bench for measurements contains a Laser displacement sensor with resolution $0.01 \mu \mathrm{m}$ and response time $100 \mu \mathrm{s}$.

The measurement starts with a ramp signal with 150 volts and the results are presented in Fig.6.

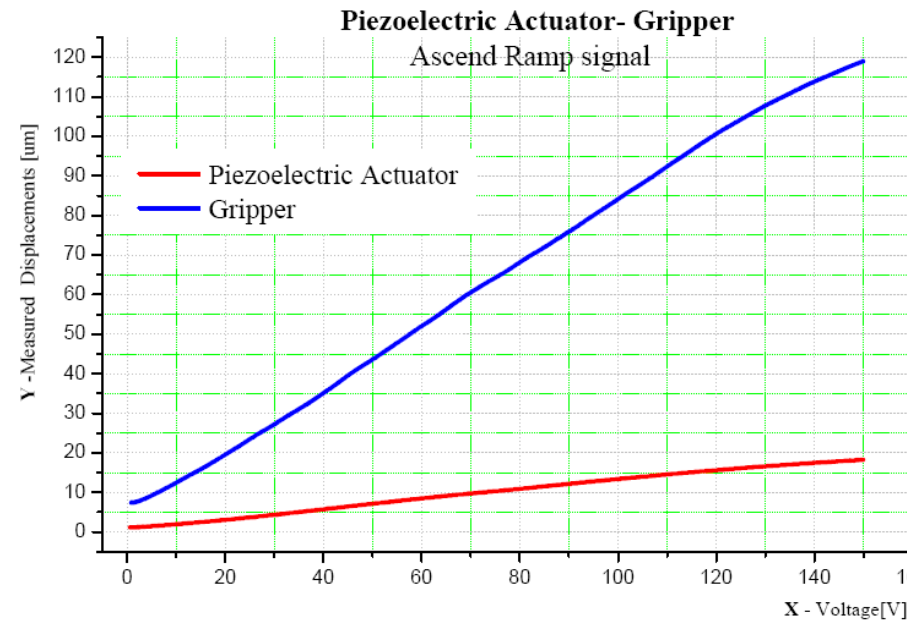

Fig. 6 The results of the measurement for ramp signal

The study was continued for the following inputs: voltage is $150 \mathrm{~V}$ and sinusoidal signal: $0.1 \mathrm{~Hz} \div 100 \mathrm{~Hz}$. The results for these measurements are presented in Fig. 7 .

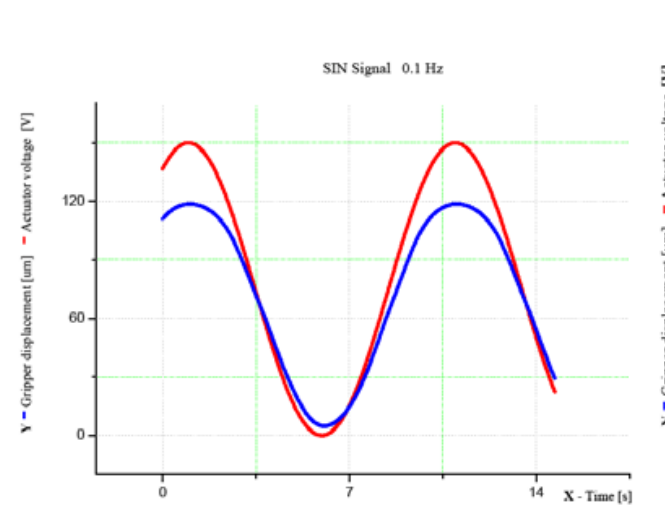

a

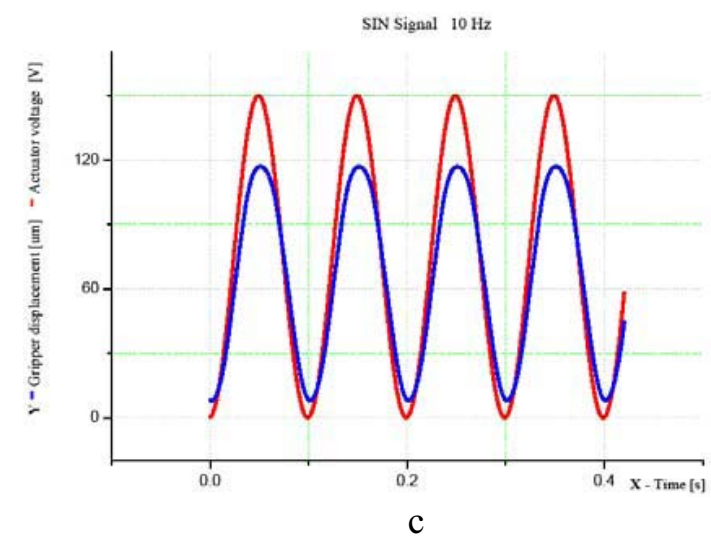

SIN Signal $1 \mathrm{~Hz}$
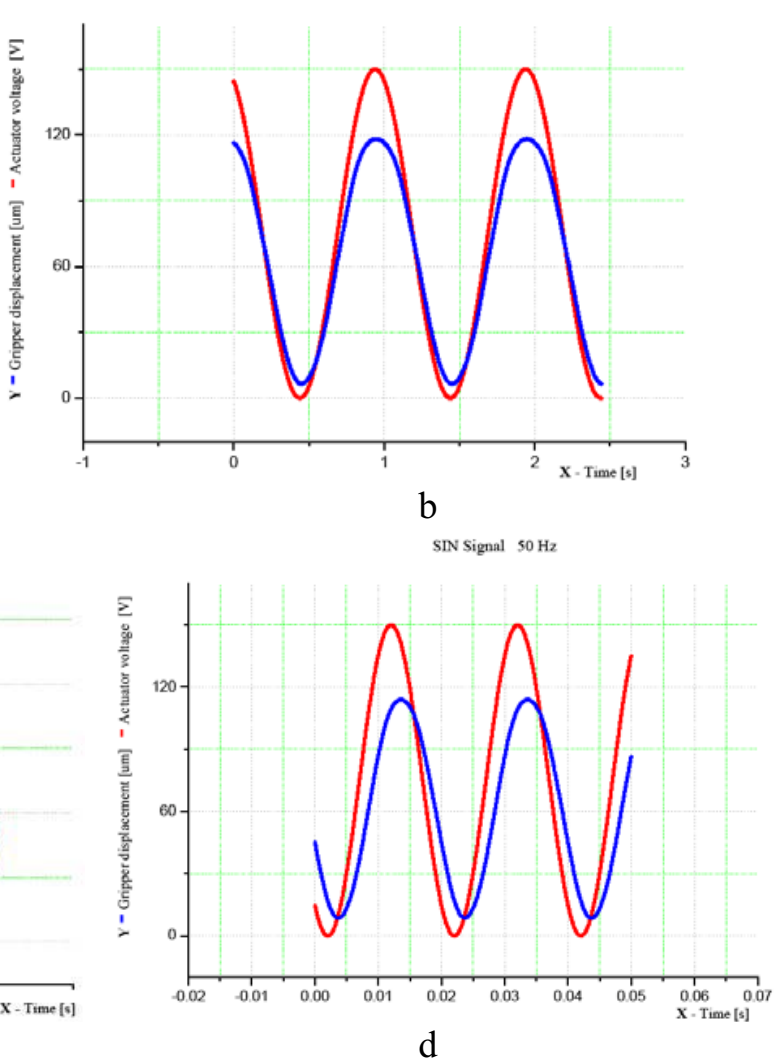

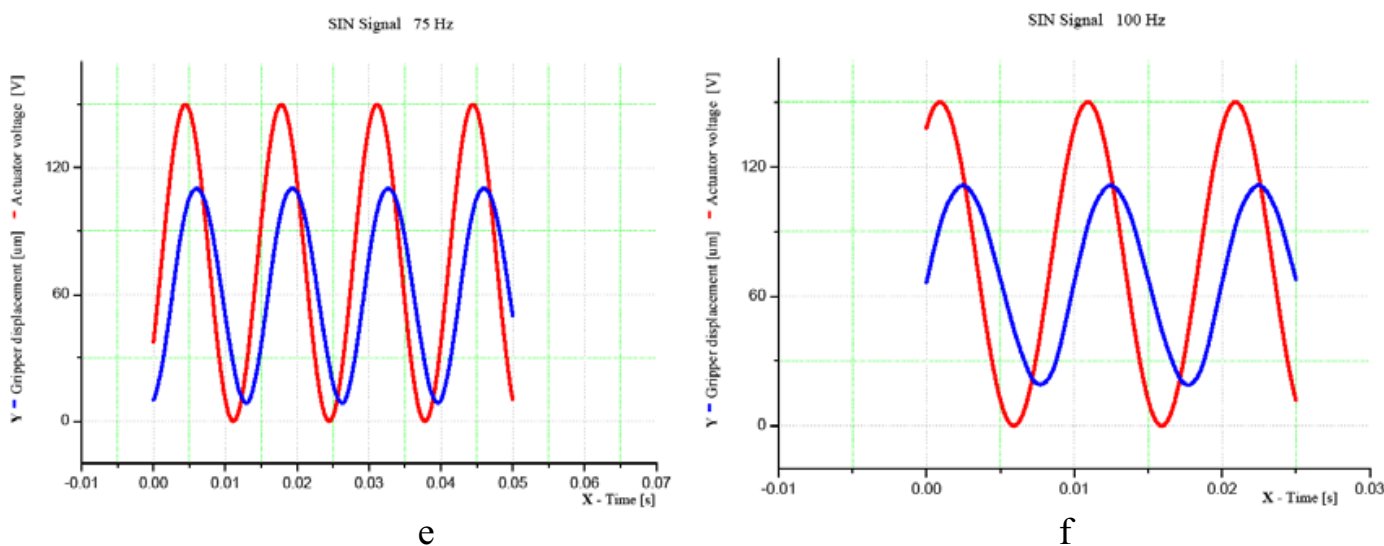

Fig. 7 The results of the measurement for sinusoidal signal

\section{Conclusion}

In this paper is presented an original mini-system witch is designed and analyzed. The structure and function of an experimental test bench is presented. The measurements were validated through the FEM analysis for ramp signal. Finite element simulations were performed in order to verify the accuracy of model-predicted compliance factors for design configuration.

The results are in good agreement for ramp signal with those produced by the analytical approach within $12 \%$ relative errors. The experimental measurements also confirm the analytical predictions with relative errors less than $8 \%$. For sinusoidal signal and frequency values higher than $10 \mathrm{~Hz}$, in the mini-system the output values of the displacement don't reach the maximum value. In conclusion, for a proper functioning the system has to be actuated with a frequency below that value.

In the future the aim of our work will be to down scale the mini-system analyzed in this paper.

\section{Acknowledgments}

The research work reported here was made possible by PNII - IDEI Project, ID 221: Modelling, Simulation and Control of the Compliant Micro mechanisms and PNII - ID 1076 Development of a Modular Family of Linear and Rotary Actuators Based on Shape Memory Alloys.

\section{References}

[1] S. Fatikow, Microsystems Technology and Microrobotics, Springer-Verlag, Berlin, (1997).

[2] D. Mândru, et al,: Actuating Systems in Precision Mechanics and Mechatronics. AlmaMater (2004)

[3] N. Lobontiu, and Garcia, E.: Mechanics of microelectromechanical systems, (Series: The Mechanical Engineering Handbook). Springer (2004)

[4] N. Lobontiu, Compliant Mechanisms: Design of Flexure Hinges, CRC Press LLC, (2002).

[5] Information on http://www.thorlabs.com

[6] S. Noveanu, Contributions concerning the study of compliant mechanisms specific to mechatronic systems, Ph.D. Thesis, (2009) 